\title{
Influence of Ferromagnetic Layer on Critical Current of a Superconducting Wire
}

\author{
F. Gömöry, M. Vojenčiak, J. Šoud And E. Seiler \\ Institute of Electrical Engineering, Centre of Excellence CENG \\ Slovak Academy of Sciences, Bratislava, Slovakia
}

\begin{abstract}
We investigated how a 10-50 $\mu$ m layer from soft ferromagnetic material, incorporated in the architecture of a composite superconducting tape, could influence the capability of the tape to transport electrical current. Numerical procedure was developed to calculate the local distribution of magnetic field and current in the condition when all the superconductor is carrying its critical current density. It was found that the concentration of magnetic flux in ferromagnetic layers allows to reduce the local value of magnetic flux density felt by the superconductor. In this way, the critical current of the tape in low applied magnetic fields can be improved. This theoretical prediction is confirmed by experimental observation.
\end{abstract}

PACS numbers: $41.20 . \mathrm{Gz}, 74.25 . \mathrm{Fy}, 74.25 . \mathrm{Nf}$

\section{Introduction}

The use of soft ferromagnetic (SF) layers as a means to improve the properties of superconducting tape conductors has been suggested by several authors [1]. However, the attempts to prepare the samples confirming this prediction failed for a long period. Now it is obvious that the shape of the layer and its incorporation into the architecture of the composite tape is of crucial importance. The kind of structure we are investigating systematically, is based on the composite multifilamentary tape from high-temperature superconductor (HTSC) $\mathrm{Bi}_{2} \mathrm{Sr}_{2} \mathrm{Ca}_{2} \mathrm{Cu}_{3} \mathrm{O}_{10}$ in $\mathrm{Ag}$ matrix (Bi-2223/Ag), the commercial standard nowadays. On the tape edges, a layer of $\mathrm{Ni}$ is deposited by electroplating in the thickness of $10-50 \mu \mathrm{m}$. Important conclusion from our previous theoretical studies [2] is that the extent, to which the tape is covered by a SF layer, should be chosen carefully. In particular, the complete cover all around the tape would reduce the critical current with respect to the original uncovered tape. In this paper, we present the results of our search for an optimal shape of the cover for a commercial HTSC tape, and demonstrate that our theoretical predictions are in agreement with experimental observations. 


\section{Sample preparation}

The HTSC tape chosen for our investigations is the Bi-2223/Ag tape produced by Trithor GmbH [3]. Its cross-section is $3.2 \times 0.25 \mathrm{~mm}^{2}$ and the critical current at $77 \mathrm{~K}$, zero applied magnetic field is $49.2 \mathrm{~A}$. To cover only its edges with $\mathrm{Ni}$, the patterned insulation was deposited in the following way: first, a layer of rubber resist was painted to the tape surface and hardened by a heat treatment. Then, a part of the resist was modified by infrared radiation, and subsequently

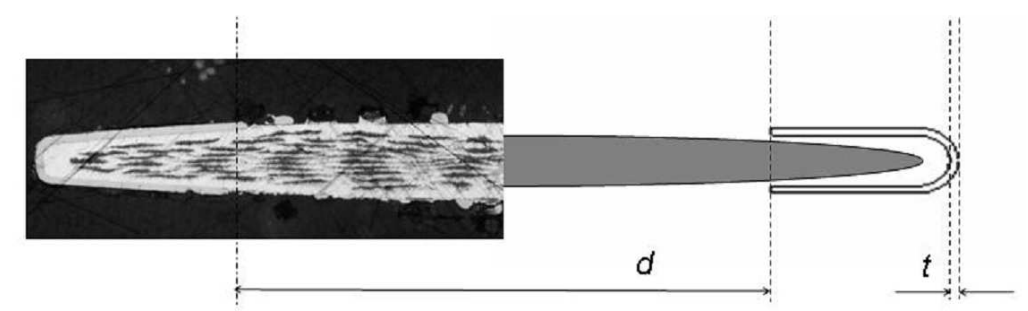

Fig. 1. Cross-section of multifilamentary tape from high-temperature superconductor Bi-2223 covered on edges by nickel (left) and its schematic representation (right). The central zone containing superconducting filaments is replaced by a superconducting ellipse. Ni-layer of thickness $t$ leaves the portion of the tape $d$ uncovered.

removed by etanol. Eventually, the tape was placed in electrolytic bath consisting in aqua solution of nickel chloride, nickel sulfate and boric acid. The remaining resist protected the covered part - in our case, it was always the central part along the tape axis, with the width $d$ - from deposition of Ni. Using different times for the deposition, thickness $t$ of the Ni-layer can be changed. In Fig. 1 there is shown the typical sample cross-section.

\section{Numerical calculation of current distribution}

Using the finite-element code Femlab [4], distributions of magnetic field and current density can be calculated for the case when all the superconductor carries the critical current density, $j_{\mathrm{c}}$. The dependence of $j_{\mathrm{c}}$ on local components of the magnetic field is taken into account using the empirical formula

$$
j_{\mathrm{c}}=\frac{J_{\mathrm{c} 0}}{\left(1+\frac{\sqrt{k_{\mathrm{B}}^{2} B_{I I}^{2}+B_{\perp}^{2}}}{B_{0}}\right)^{\beta}},
$$

where the parameters $j_{\mathrm{c} 0}, B_{0}, k_{\mathrm{a}}$, and $\beta$ characterise the properties of an effective superconducting medium replacing the superconductor-metal composite of the central multifilamentary zone. These parameters have been found by comparing the values of critical current, $I_{\mathrm{c}}$, measured on the original bare tape, with numerical simulations. Using $\approx 15$ data points for both the normal and parallel magnetic field, the agreement characterized by the standard deviation $<0.2 \mathrm{~A}$ 
has been achieved using the following values: $j_{\mathrm{c} 0}=1.92 \times 10^{8} \mathrm{~A} / \mathrm{m}^{2}, k_{\mathrm{a}}=0.12$, $B_{0}=0.0037 \mathrm{~T}, \beta=0.47$. To know the properties of Ni-cover, the sample was prepared by depositing $10 \mathrm{~m}$ Ni-layer on a $1 \mathrm{~mm}$ thick $\mathrm{Ag}$-sheet with the same procedure as used for the superconducting tapes. Magnetisation data in parallel field can be satisfactorily described by the fit

$$
\mu_{r}=1+\frac{\mu_{r \max }}{1+\left(B^{2} / B_{\mathrm{c}}^{2}\right)^{\alpha}}
$$

with parameters $\mu_{r \max }=250, B_{\mathrm{c}}=0.32 \mathrm{~T}$, and $\alpha=1.7$. With these parameters, the finite-element code can resolve the distribution of current density and local magnetic field inside the superconducting zone. Results of these calculations are plotted in Fig. 2 for $30 \mu \mathrm{m}$ thick Ni layer. Darker colour means higher current density. It is obvious that by placing the Ni-cover around the tape edges, a large central portion of superconductor is exposed to a lower magnetic field or oriented
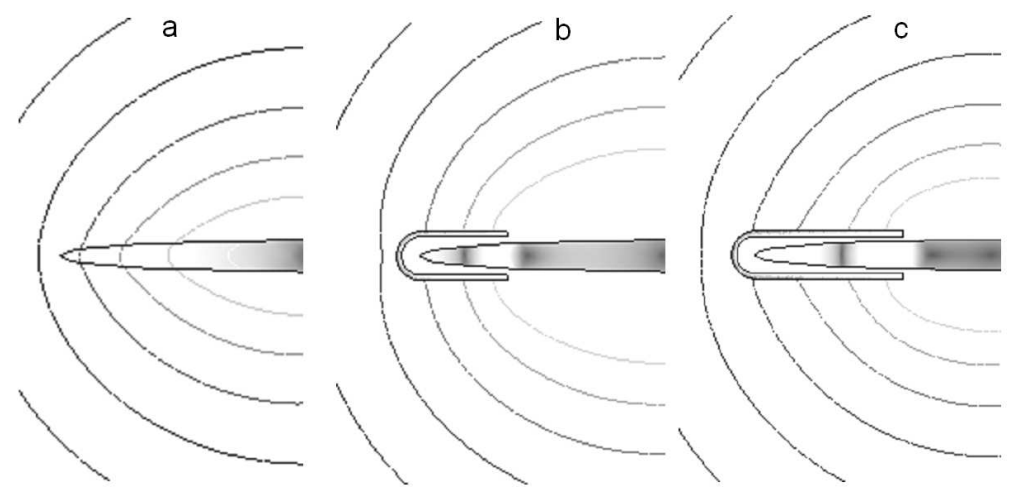

Fig. 2. Calculated distribution of current density (grey scale) and magnetic field (lines of constant vector potential) for the original tape (a) and two tapes covered by Ni-layer. No additional magnetic field is applied to the tape (self-field conditions).

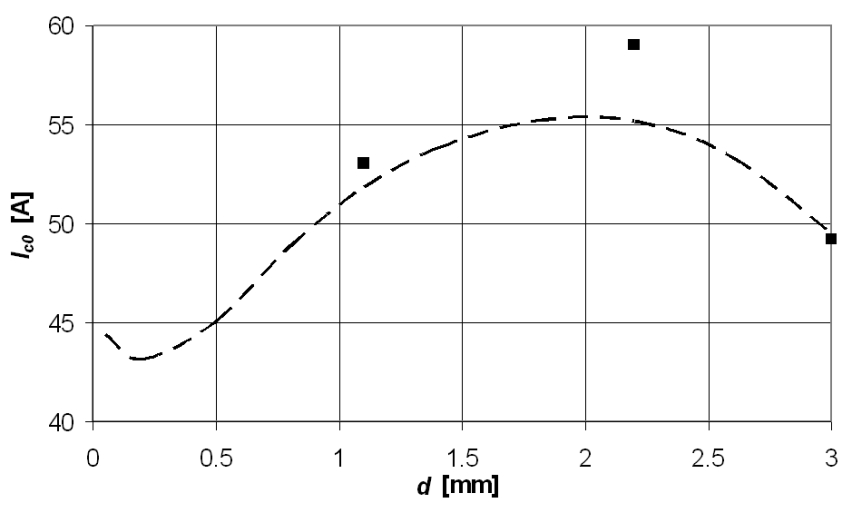

Fig. 3. Dependence of the self-field critical current, $I_{\mathrm{c} 0}$, on the width uncovered by Ni. Dashed line - theoretical prediction, squares - experimental results. 
more in favourable direction. From the calculated distributions one can find the critical current by integrating the current density across the superconducting area. Repeating the calculation for different values of the parameter $d$, we have discovered that there is an optimum in the extent of the Ni-cover — see Fig. 3. All the information gathered in this figure regards the Ni-layer of $30 \mu \mathrm{m}$ thickness. Together with theoretical predictions obtained by the finite element code calculations, three experimental points representing the properties of samples with the geometries given in Fig. 2 are inserted. Qualitative agreement is satisfactory, however a further refinement of the calculation method is necessary to achieve a more precise prediction.

\section{Acknowledgments}

This work was in part financially supported by the Slovak Research and Development Agency under contract No. APVV- 51-045605.

\section{References}

[1] M. Majoros, B.A. Glowacki, A.M. Campbell, Physica C 338, 251 (2001).

[2] F. Gömöry, Appl. Phys. Lett. 89, 072506 (2006).

[3] http://www.trithor.com/pdf/datasheets/Trithor_HTSWires_w.pdf.

[4] FEMLAB 3.0 Electromagnetics Module User's Guide, Comsol AB 1994-2004, http://www.comsol.com/. 